Article

\title{
Optimal Design of Electromagnetically Actuated MEMS Cantilevers
}

\author{
Paolo Di Barba ${ }^{1}$, Teodor Gotszalk ${ }^{2}$, Wojciech Majstrzyk ${ }^{2}$ (D), Maria Evelina Mognaschi ${ }^{1, *}$ (1), \\ Karolina Orłowska ${ }^{2}$, Sławomir Wiak ${ }^{3}$ and Andrzej Sierakowski ${ }^{4}$ \\ 1 Department of Electrical, Computer and Biomedical Engineering, University of Pavia, I-27100 Pavia, Italy; \\ paolo.dibarba@unipv.it \\ 2 Faculty of Microsystem, Electronics and Photonics, Wrocław University of Science and Technology, \\ PL-50372 Wrocław, Poland; teodor.gotszalk@pwr.edu.pl (T.G.); wojciech.majstrzyk@pwr.edu.pl (W.M.); \\ karolina.orlowska@pwr.edu.pl (K.O.) \\ 3 Institute of Mechatronics and Information Systems, Łódź University of Technology, PL-90924 Łódź, Poland; \\ slawomir.wiak@p.lodz.pl \\ 4 Institute of Electron Technology, PL-02668 Warsaw, Poland; asierak@ite.waw.pl \\ * Correspondence: eve.mognaschi@unipv.it; Tel.: +39-0382-985-785
}

Received: 29 June 2018; Accepted: 30 July 2018; Published: 2 August 2018

check for updates

\begin{abstract}
In this paper we present the numerical and experimental results of a design optimization of electromagnetic cantilevers. In particular, a cost-effective technique of evolutionary computing enabling the simultaneous minimization of multiple criteria is applied. A set of optimal solutions are subsequently fabricated and measured. The designed cantilevers are fabricated in arrays, which makes the comparison and measurements of the sensor properties reliable. The microfabrication process, based on the silicon on insulator (SOI) technology, is proposed in order to minimize parasitic phenomena and enable efficient electromagnetic actuation. Measurements on the fabricated prototypes assessed the proposed methodological approach.
\end{abstract}

Keywords: electromagnetically actuated cantilevers; nanometrology; multiobjective optimization; active cantilevers; SOI-based prototyping

\section{Introduction}

Micro-electromechanical systems (MEMS) are micromachines containing movable parts whose deflection is controlled and detected electronically. In general MEMS are manufactured using technologies applied in microelectronics. In this way fabrication of devices of various functions and properties can be done in a batch process, which increases repeatability and system reliability.

The critical physical dimensions of MEMS devices, to which belong simple structures having no moving elements and extremely complex electromechanical systems with elements actuated and controlled by the integrated microelectronics, can vary from several microns to several millimeters. In a natural way the MEMS technology merges at the nanoscale into nano-electromechanical systems (NEMS). Among others, the so called supported cantilevers, whose elasticity can be described with relatively high accuracy using simple models, are MEMS devices. Moreover, the variety of possible applications is very broad, ranging from scanning probe microscopy (SPM) to sensing systems applied in biochemistry and biotechnology [1]. In all the aforementioned applications the cantilever static or resonance deflection is detected to follow the phenomena of interest. Optical and electrical techniques, which can be applied in single or array cantilever (sensor) operation, enable monitoring of thermomechanical noise structure vibration. However, it should be mentioned that the full interoperability of the cantilever system can only be obtained when the structure deflection is 
electrically controlled. This means that the deflection should be maintained in the feedback loop by a deflection actuator, which is integrated with the movable mechanical part. This way, as the mass of the cantilever is quite small, the actuator induces device movement with the highest energetic efficiency and speed. Moreover, the actuation reliability is improved as the deflection is actuated of only the movable part. There are various electrical technologies for actuation of the cantilever displacement. The application of electrostatic actuation scheme is usually limited to the cantilevers of big dimensions [2]. Moreover, it involves electrode biasing with relatively high voltage which cannot often be applied in MEMS technology.

The cantilever deflection can be controlled piezoelectrically as well [3]. In this solution a piezoelectrical thin film is deposited on the cantilever and when it is electrically biased the structure displacement can be excited. Similar to the electrostatic technology, the actuator bias voltage is quite high which hinders the applications in the integrated sensing systems. Moreover, deposition of the piezoelectric thin film is cumbersome and its repeatability is often limited. From the fabrication point of view, the electrothermal actuator technology is significantly simpler [4]. In this case, a spring beam integrates a microheater dissipating heat due to Joule effect. As a consequence of different thermal expansion coefficients of materials, forming the entire structure, mechanical stress occurs in the movable part [5]. As a result the structure deflects. In this way the deflection in the frequency range of up to several $\mathrm{MHz}$ can be induced [6]. In the DC regime the cantilever can be deflected by even few micrometers [4]. The drawback of the electrothermal actuation scheme is that its application in liquids and consequently in biochemistry and biotechnology is limited. In this case, the heat generated in the microheater can not only propagate through the beam structure itself, but also in the surrounding liquid, reducing significantly the actuation efficiency. Moreover, in the electrothermal scheme the cantilever deflection can be controlled in one direction only.

In contrast to that, the electromagnetic actuation technology is free from aforementioned drawbacks. In this approach the cantilever integrates a conductive loop called Lorentz loop. When the electromagnetic cantilever is electrically biased and immersed in the magnetic field, the electromagnetic force induces deflection $[6,7]$.

The bidirectional cantilever deflection can be analysed based on the model describing the electromagnetic force in Lorentz loop. The actuation force can be computed by means of the Lorentz force equation. The strong magnetic field in the range of fractions of a tesla can be excited by external magnets. The current in the loop can be controlled by the low voltage electronics and the length of Lorentz loop can be determined with high accuracy. In this way DC and AC displacement can be controlled with the highest precision, efficiency and reliability. Moreover, it is relatively straight forward to design systems operating at very low energy integrated with Application Specific Integrated Circuits (ASICs). The electromagnetic cantilever was introduced for the first time by Shen [8]. At the resonance, the cantilevers can be observed with high resolution, hence they were also applied as high resolution magnetic field sensors [9,10] and resonators [11].

The actuation precision and reliability were the main reasons, why the electromagnetic cantilevers were successfully applied in SPM [12,13]. In all these investigations the cantilever deflection was determined metrologically (in other words quantitatively), which is of significant importance for investigations of the interactions at a scanning probe microscopy tip.

The electromagnetic cantilevers were also utilized in metrology of the mechanical stress associated with the adsorbtion of molecular self-assembled layers (SAMs) on the gold cantilever surface. When thiol molecules adsorb (covalently bind) on the cantilever surface, mechanical stress occurs between molecular film and the cantilever leading to the structure deflection. Such a response was usually used to detect (indicate qualitatively) chemical surface reactions with high resolution [14].

In our experiments the cantilever deflection caused by the molecular adsorbtion was observed optically and the electromagnetic force was applied to compensate the structure deflection. As the electromagnetic force was determined precisely by the bias current, the magnetic field and the 
Lorentz loop dimensions, the electro-mechanical phenomena were described quantitatively for the first time [15].

Despite the many advantages of electromagnetic actuation, its limitations must be identified as well. To the most important problems, which must be taken into account, belongs parasitic thermomechanical structure actuation when Lorentz loop is electrically biased. In this case, due to various coefficients of linear extension of the materials forming the spring beam, heat dissipated in the structure leads to additional structure deflection.

The spring beam should exhibit low stiffness in order to control the structure deflection in the range of up to several micrometers, which additionally correlates with higher force and mass change detection resolution. Moreover, the resonance frequency of the designed and fabricated electromagnetic structures must be as high as possible, which makes it insensitive to the measurement disturbances and decreases the time response. The low stiffness and high resonance frequency can be achieved when the length and the thickness of the cantilever are reduced in the appropriate way. However, in the case of the electromagnetic cantilevers one should also optimize the structure geometry in order to reduce Lorentz loop resistance. In this way the heat dissipated in the biased beam and the parasitic thermomechanical actuation are significantly reduced. Unfortunately, simple analysis of the equations modelling the electromagnetic cantilevers does not allow a closed-form design solution and only the optimal design methods make it possible to overcome the abovementioned limitations. In general, the methods of automated optimal design are based on repeated analysis to solve the field model [16], which ultimately influence the computational budget of the simulation.

Against this background, the available technology makes it possible to fabricate electromagnetically actuated cantilevers with no severe limitation on the shape. On the other hand, high accuracy measurements demand low stiffness, low power losses and high resonance frequency. Hence, the exploitation of methods for automated optimal design help to achieve these requirements with subsequent prototyping of better performing cantilever. A contribution towards this goal is here presented. Specifically, the paper is organized as follows: in Sections 2.1 and 2.2 forward and inverse models describing the behavior of the cantilever are presented. In Section 2.3 the fabrication process is described. In Sections 3.1-3.3 the optimization results are presented, while in Sections 3.4 and 3.5 the measurement results are shown. Finally, a discussion is presented and conclusions are drawn.

\section{Materials and Methods}

The deflection of the MEMS device can be induced in the electromagnetic way. In this technology external magnetic field interacts with the current flowing through conductive loop (Figure 1) producing a Lorentz force $\mathbf{F}_{\mathbf{z}}[6,7]$.
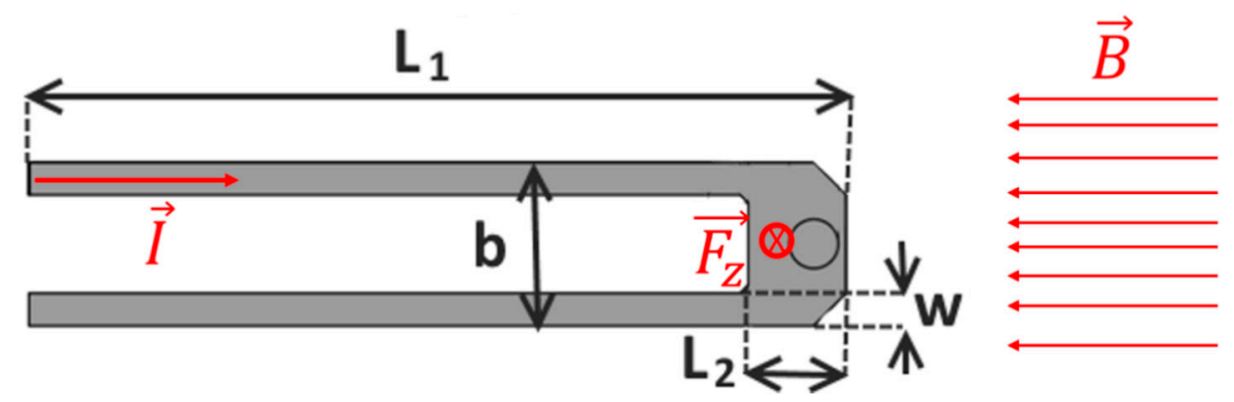

Figure 1. Design variables of the cantilever (black symbols) and schematic representation of the electromagnetic actuation by means of the Lorentz force (red lines).

\subsection{Optimal Design of an Electromagnetically Actuated Cantilever: Direct Problem}

The direct (or analysis) problem reads as follows given the shape $\mathbf{g}$ of the cantilever end, current $\mathbf{I}$, and magnetic induction $\mathbf{B}$, find: 
the stiffness $k$ of the cantilever;

the resonance frequency $f$ of the cantilever;

the force $F_{z}$ acting on the end region and its displacement $\Delta z$;

the electric resistance $R$ (power-loss related) of the Lorentz loop.

The stiffness $k$ of the cantilever can be calculated [17] as follows:

$$
k=\frac{E b w t^{3}}{2 b\left(L_{1}^{3}-L_{2}^{3}\right)+4 w L_{2}^{3}}\left[\mathrm{Nm}^{-1}\right]
$$

where $E$ is the Young's modulus, $b$ is the cantilever width, $w$ the arm width, $t$ is the thickness equal to $1.5 \mu \mathrm{m}, L_{1}$ is the cantilever length and $L_{2}$ is the tip length (see Figure 1).

The resonance frequency $f$ can be evaluated with the following approximate formula [18]:

$$
f \cong 0.161 \frac{t}{L_{1}^{2}} \sqrt{\frac{E}{\rho}}\left[\mathrm{rad} \mathrm{s}^{-1}\right],
$$

where $\rho$ is the mass density equal to $2330 \mathrm{kgm}^{-3}$.

The force $F_{z}$ and its displacement $\Delta z$ can be calculated as follows:

$$
\begin{aligned}
& F_{z}=I b B, \\
& \Delta z=\frac{F_{z}}{k} .
\end{aligned}
$$

Equation (3), which is derived from the Lorentz's equation, is under the assumption that the cantilever, i.e., the plane in which the current flows, is perpendicular to the magnetic induction field.

Finally, the electric resistance $R$ can be calculated as the series of three electric resistances of the three path components (two arms, with the same resistance value $R_{1}$ and the tip, with resistance $R_{2}$ ):

$$
R=2 R_{1}+R_{2} \cong 2 \frac{\sigma^{-1}\left(L_{1}-L_{2}\right)}{w t}+\frac{\sigma^{-1} b}{L_{2} t}
$$

where $\sigma$ is the electric conductivity of the boron-doped silicon (without metal layer) equal to $6.67 \times 10^{4} \mathrm{Sm}^{-1}$.

\subsection{Optimal Design of an Electromagnetically Actuated Cantilever: Inverse Problem}

If the shape of the cantilever end is defined by means of a $n$-dimensional vector $g=\left(g_{1}, \ldots\right.$, $g_{k}, \ldots, g_{n}$ ) of geometric variables (e.g., for a polygonally-shaped end region, the coordinates of the relevant vertices), the inverse (or design) problem reads: given current $I$ and magnetic induction $B$, find the shape $g=\left(g_{1}, \ldots, g_{k}, \ldots, g_{n}\right)$ of the cantilever end region such that:

the stiffness $k(g)$ of the cantilever is minimized;

the resonance frequency $f(g)$ is maximized;

the displacement $\Delta z(g)$ of the end region is maximized;

the electric resistance $R(g)$ of the Lorentz loop is minimized.

A multi-objective optimization problem characterized by four objective functions $[k(g), f(g), \Delta z(g)$, $R(g)$ ] is originated. When more than one objective function is considered in the optimization, more solutions, belonging to the so-called Pareto front, are obtained. In particular, a solution is called Pareto optimal if there does not exist another solution that dominates it i.e., a solution that cannot be improved in any of the objectives without degrading at least one of the other objectives.

Considering $n$-objective functions, a solution $g_{1}$ is said to dominate another solution $g_{2}$, if:

$$
f_{i}\left(g_{1}\right) \leq f_{i}\left(g_{2}\right) \forall i \in(1, n) \text { and }
$$




$$
\exists j \in(1, n) \text { such that } f_{j}\left(g_{1}\right)<f_{j}\left(g_{2}\right) .
$$

A solution $g_{1}$ is called Pareto indifferent with respect to a solution $g_{2}$ if:

$$
\begin{gathered}
\exists j \in(1, n) \text { such that } f_{j}\left(g_{1}\right)<f_{j}\left(g_{2}\right) \text { and } \\
f_{i}\left(g_{1}\right) \leq f_{i}\left(g_{2}\right) \forall i \neq j \in(1, n)
\end{gathered}
$$

In our inverse problem it turns out to be $n=4$ and $f_{1}=k(g), f_{2}=f(g), f_{3}=\Delta z(g)$ and $f_{4}=R(g)$. Our goal is, starting from a prototype geometry $g_{0}$, to find a new geometry improving $g_{0}$ against the four objectives, according to Equations (6)-(9).

When many objective functions (say more than two) are considered, it is very common to find solutions of the optimization problem which are indifferent in the Pareto sense to the starting point. However, these solutions are nevertheless interesting because they improve at least one objective function.

It can be noted that Equations (1)-(5) define an analytical model for the direct problem, however, Equations (6)-(9) prevent from an analytical solution of the inverse problem and therefore the numerical method is in order.

The shape of the cantilever is defined by four design variables, as shown in Figure 1:

$w$, arm width.

$L_{1}$, cantilever length.

$L_{2}$, tip length.

$b$, cantilever width.

The variation range for each design variable is shown in Table 1 . The chosen values for the boundaries are based on the experience.

Table 1. Variation range for the design variables (units in $\mu \mathrm{m}$ ).

\begin{tabular}{lcccc}
\hline & $\boldsymbol{w}$ & $\boldsymbol{L}_{\mathbf{1}}$ & $\boldsymbol{L}_{\mathbf{1}}$ & $\boldsymbol{b}$ \\
\hline Lower bound & 20 & 100 & 50 & 100 \\
Upper bound & - & 600 & 100 & 150 \\
\hline
\end{tabular}

In order to guarantee a geometrical congruency, the following constraint (units in $\mu \mathrm{m}$ ) is set:

$$
b \geq 2 w+10 \text {. }
$$

A series of optimizations are subsequently run, considering one (Opt1), two (Opt2) or three (Opt3) objective functions at a time:

Opt1-each objective function i.e., $k, f, \Delta z$ and $R$, is individually optimized in four different single-objective optimizations (Opt1k, Opt1f, Opt1z, Opt1R). In each case, the activated objective function is the leading one, while the remaining three objective functions are updated, depending on the current value of the design vector $\mathrm{g}$;

Opt2 - the following optimizations are run: $k$ and $R$ are optimized (Opt2kR), $f$ and $z$ are optimized (Opt2fz), $f$ and $R$ are optimized (Opt2fR), $z$ and $R$ are optimized (Opt2zR). Each pair of objective function is optimized in the Pareto sense, the remaining two functions are updated, depending on the current value of the design vector $g$;

Opt3 $-k, z$ and $R$ are optimized (Opt3) in the Pareto sense, while the frequency $f(g)$ is simply updated.

In order to solve these optimization problems, an evolutionary algorithm of lowest order is applied [19]. This algorithm is able to solve single-objective problems (in this case it is called "ESTRA 
method" [20,21]) and multi-objective problems ("MOESTRA method" [22]). The search in the design space begins in a region of radius $\mathbf{d}_{0}$ (standard deviation) centered at the initial point $\mathbf{m}_{\mathbf{0}}$ (mean value); $\mathbf{m}_{\mathbf{0}}$ is externally provided, while $\mathbf{d}_{\mathbf{0}}$ is internally calculated on the basis of the bounds boxing the variation of the design variables.

Setting $\mathbf{m}=\mathbf{m}_{\mathbf{0}}$ and $\mathbf{d}=\mathbf{d}_{\mathbf{0}}$, the generation of the design vector $\mathbf{x}=\mathbf{m}+\mathbf{u} \mathbf{d}$ then proceeds, resorting to a normal sample $\mathbf{u} \in(0,1)$. It is verified that $x$ fulfils bounds and constraints (i.e., that $x$ is feasible), otherwise a new design vector is generated until it falls inside the feasible region.

The associated objective function $f(\mathbf{x})$ is then evaluated and the test if $f(\mathbf{x})$ dominates $f(\mathbf{m})$ (Equations (6) and (7)) is performed; if the test is successful, $\mathbf{m}$ is replaced by $\mathbf{x}$ (the so-called selection process), otherwise $\mathbf{m}$ is retained.

The next step is concerned with the size of the search region that will be used for the successive iterations. The underlying rationale is that when a point better than the current one is found, the radius of the search region is increased around the new point to search for further improvements; if no improvement is found, the radius of the search region is gradually decreased up to convergence (annealing process).

In this respect, the evolutionary algorithm substantially differs from a deterministic one e.g., Nelder and Mead algorithm [23], in which the search region would be narrowed around the better point in order to converge towards the corresponding, nearest minimum. The drawback is that this minimum might be a local one. On the contrary, the evolutionary algorithm, if successful in finding a better point, covers a larger region of search in order to see if there would be another good candidate in the neighborhood, and then does the opposite when this is not deemed possible. This way, there is a non-zero probability of finding the region where the global optimum of the objective function is located. To assess the optimization results, a set of prototypes has been fabricated based on the technology described in the subsequent Section.

\subsection{Fabrication Process}

The fabrication process of the microcantilevers used for a radiation pressure sensing was based on a double side micromachining concept [24]. However, in contrast to the typical technology based on bulk silicon substrates, in this case the silicon on insulator (SOI) wafers with 1 and 1.5 micrometers thick buried oxide and cantilever layer, respectively, were used as the input material. Despite the fact that the use of SOI substrates is more expensive, this solution has many advantages compared to the use of the bulk wafers. Two advantages of using the SOI substrates are particularly important.

The first advantage is a significant simplification of the microcantilever production process. The second advantage is a guarantee that all cantilevers defined on one wafer are characterized by uniform thickness regardless of its shape and size (the thickness depends only on the SOI wafer cantilever layer properties).

Therefore, using the SOI substrate, the production technology consists of only four technological steps: high $\mathrm{p}$ doping of the whole cantilever layer, definition of a gold contacts and mirrors, definition of the shape of the cantilever and finally the releasing of the cantilevers. Figure 2 presents the scanning electron microscopy (SEM) image of the cantilever after three steps, i.e., after plasma etching processes. Upon magnification (Figure 2b,c) the four layers can be observed: gold (which serves as a mirror), a $1.5 \mu \mathrm{m}$ silicon layer, a buried silicon dioxide and a handle silicon wafer. The example final cantilevers matrix after released operation is presented in Figure 3.

The presented construction was optimized for the electromagnetic actuation, first proposed by Buguin group [25]. The use of SOI wafer makes it easy to obtain a homogeneous doping of the cantilever defined in the SOI cantilever layer, which significantly reduced the thermal actuation effect.

The homogeneous high-p-doped cantilever layer was obtained by using boron doped layer deposited by Low Pressure Chemical Vapor Deposition (LPCVD) method [26]. In this method there are two technological stages: boron source layer deposition using $\mathrm{B}_{2} \mathrm{H}_{6} 5 \%$ in $\mathrm{N}_{2}$ at $270{ }^{\circ} \mathrm{C}$ (pre-diffusion) and high temperature annealing (diffusion of the boron dopant from previously deposited layer into 
silicon). The boron dopant profile can be optimized by controlling the duration time of pre-diffusion and duration time and temperature of the diffusion step.

By changing the pre-diffusion time we can control the surface boron concentration. In our experiments, the duration of the pre-diffusion was $55 \mathrm{~min}$. Relatively long times allow one to obtain a high boron concentration of $1 \times 10^{20} \mathrm{~cm}^{-3}$.

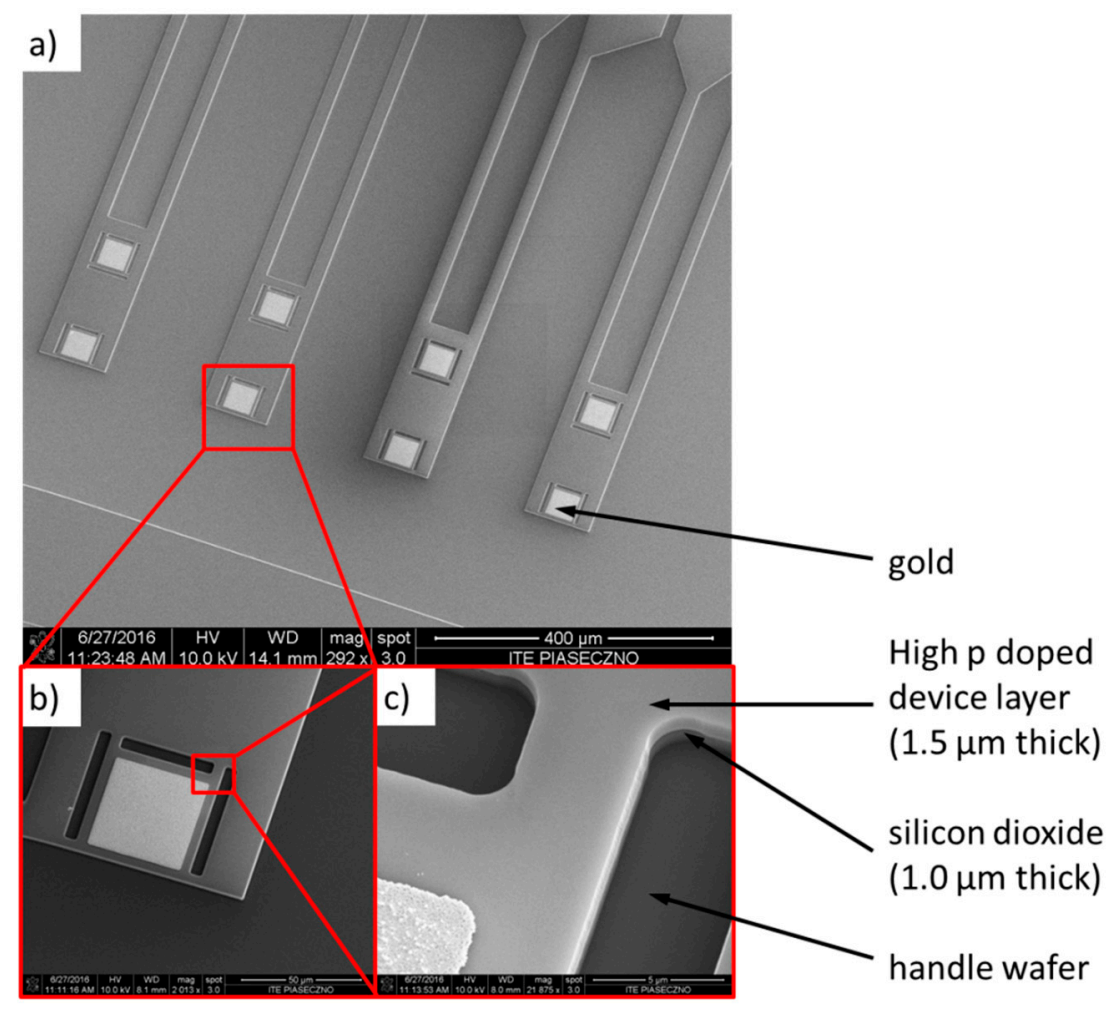

Figure 2. The scanning electron microscope (SEM) image of the cantilever matrix after three technological steps (inter-operative control).

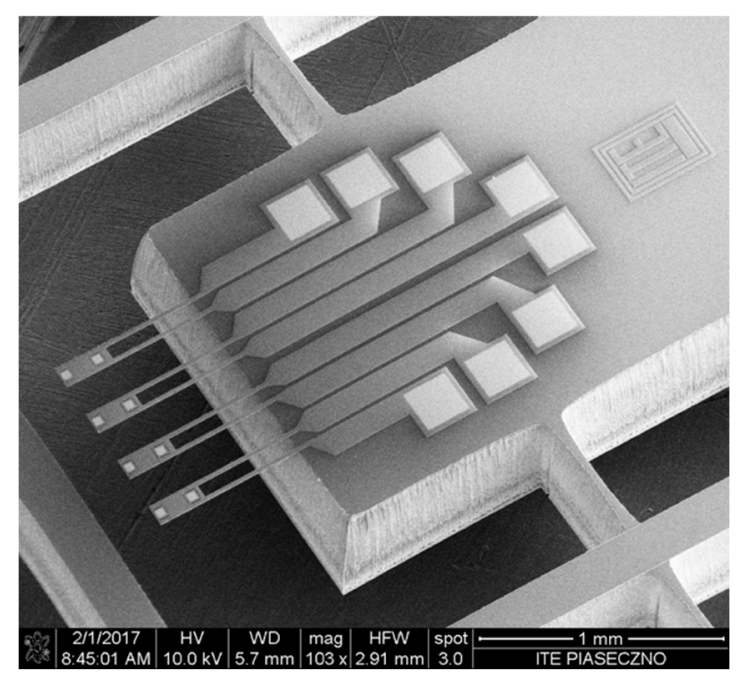

Figure 3. The scanning electron microscope (SEM) image of the final structure. Scanning parameters of the image. High Voltage $(\mathrm{HV})=10 \mathrm{kV}$; Working Distance $(\mathrm{WD})=5.7 \mathrm{~mm}$; Horizontal Field of View $(\mathrm{HFW})=2.91 \mathrm{~mm}$. 
On the other hand, changing the temperature and duration time of diffusion allows one to control the final dopant profile in the device layer. In our case the temperature and duration time of the diffusion were $1100{ }^{\circ} \mathrm{C}$ and $25 \mathrm{~min}$, respectively. Further increases in value of these parameters would cause an undesirable effect of reducing the surface boron concentration. In this case the dopant profile would not be homogeneous and would increase the thermal actuation effect. On the other hand, decreasing the diffusion temperature causes incomplete oxidation of the layer used as a source of boron, increasing its etch resistance, and makes its removal difficult.

The results of simulation of the obtained dopant profile for the listed above process parameters are presented in Figure 4. The simulation confirmed that the boron dopant profile is uniform along the cantilever layer thickness; thus, the thermal expansion coefficient should be also constant [27].

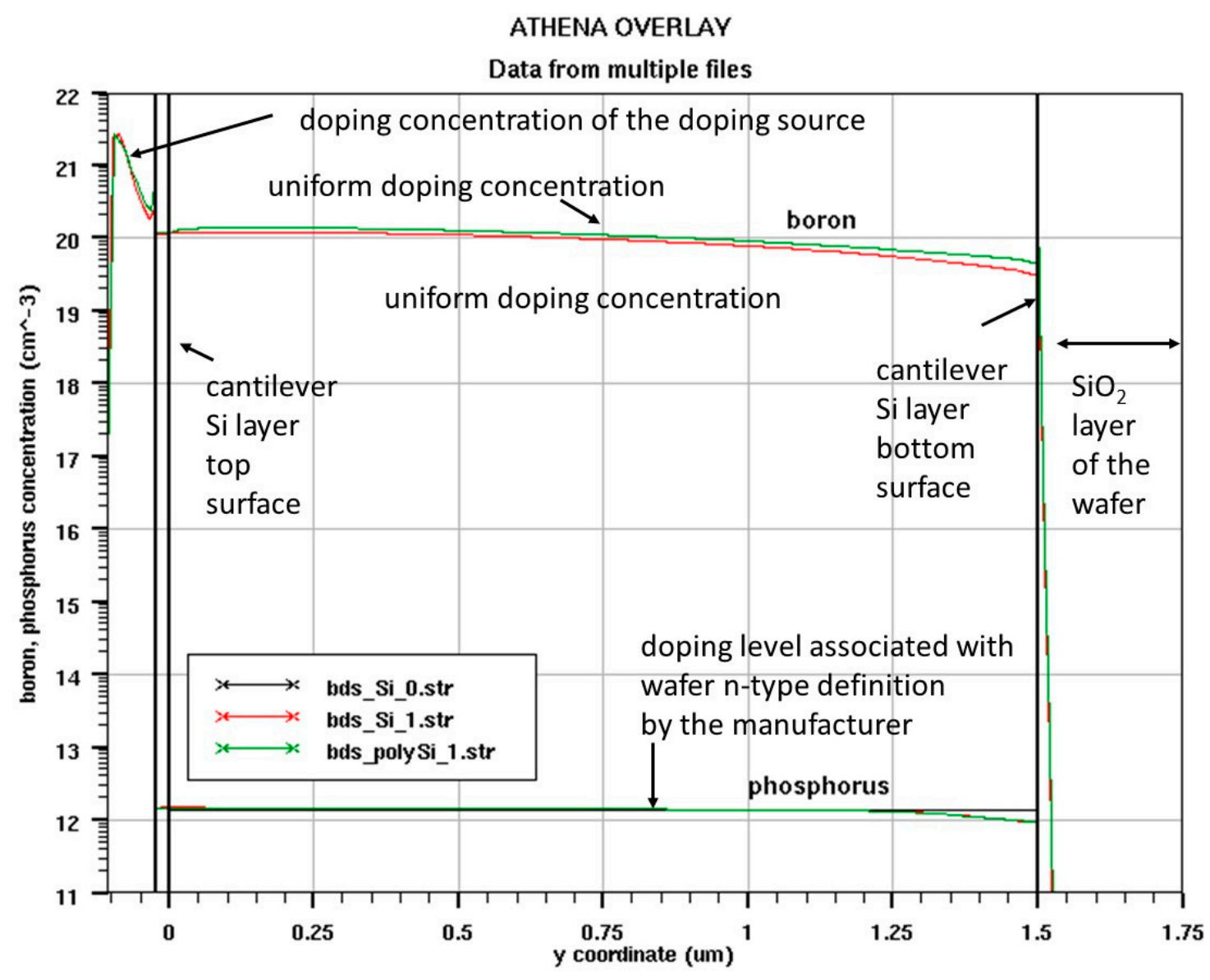

Figure 4. The high concentration of $\mathrm{p}$ dopant layer simulation result for the silicon on insulator (SOI) cantilever. Red and green curves represent two methods of doping concentration calculation.

\section{Results}

\subsection{Single-Objective Optimization Results}

The results of the single-objective optimizations are shown in Table 2. In each table the values of the design variables and of the functions $(k, f, \Delta z, R)$ are shown. In particular, the minimized objective function is highlighted in bold. 
Table 2. Single-objective optimization Opt1k results.

\begin{tabular}{ccccccccc}
\hline & $\boldsymbol{w}[\boldsymbol{\mu \mathrm { m }}]$ & $\boldsymbol{L}_{\mathbf{1}}[\boldsymbol{\mu \mathrm { m }}]$ & $\boldsymbol{L}_{\mathbf{2}}[\boldsymbol{\mu \mathrm { m }}]$ & $\boldsymbol{b}[\boldsymbol{\mu \mathrm { m }}]$ & $\boldsymbol{k}\left[\mathbf{N m}^{-\mathbf{1}}\right]$ & $f[\mathbf{k H z}]$ & $\boldsymbol{\Delta z}[\mathrm{nm}]$ & $\boldsymbol{R}[\Omega]$ \\
\hline Initial & 20.0 & 500 & 50.0 & 100 & $4.32 \times 10^{-2}$ & 8.00 & 925 & 470 \\
Opt1k & 21.1 & 569 & 53.0 & 114 & $\mathbf{3 . 0 9} \times \mathbf{1 0}^{-\mathbf{2}}$ & 6.18 & 1482 & 512 \\
Opt1f & 24.6 & 210 & 64.6 & 123 & 0.726 & $\mathbf{4 5 . 3}$ & 67.6 & 138 \\
Opt1z & 22.0 & 568 & 63.9 & 128 & $3.25 \times 10^{-2}$ & 6.21 & $\mathbf{1 5 7 4}$ & 478 \\
Opt1R & 47.6 & 211 & 80.7 & 119 & 1.39 & 45.2 & 34.3 & $\mathbf{6 9 . 3}$ \\
\hline
\end{tabular}

From Table 2-Opt1k, it can be noted that the non-controlled objective functions $f$ decreases, which is undesirable, $\Delta z$ increases (desirable) and $R$ increases (undesirable).

From Table 2-Opt1f, it can be noted that the non-controlled objective functions $k$ increases, which is undesirable, $\Delta z$ decreases (undesirable) and $R$ decreases (desirable).

From Table 2-Opt1z, it can be noted that the non-controlled objective functions $k$ decreases (desirable), $f$ decreases (undesirable) and $R$ increases (undesirable).

From Table 2-Opt1R, it can be noted that the non-controlled objective functions $k$ increases (undesirable), $f$ increases (desirable) and $\Delta z$ decreases (undesirable).

\subsection{Bi-Objective Optimization Results}

The results of the bi-objective optimizations are shown in Table 3. In each table the values of the design variables and of the functions $(k, f, \Delta z, R)$ are shown. In particular, the minimized objective functions are highlighted in bold.

Table 3. Bi-objective optimization results.

\begin{tabular}{ccccccccc}
\hline & $w[\boldsymbol{\mu m}]$ & $\boldsymbol{L}_{\mathbf{1}}[\boldsymbol{\mu \mathrm { m }}]$ & $\boldsymbol{L}_{\mathbf{2}}[\boldsymbol{\mu \mathrm { m }}]$ & $\boldsymbol{b}[\boldsymbol{\mu \mathrm { m } ]}$ & $\boldsymbol{k}\left[\mathbf{N m}^{-\mathbf{1}}\right]$ & $f[\mathrm{kHz}]$ & $\boldsymbol{\Delta z}[\mathrm{nm}]$ & $\boldsymbol{R}[\Omega]$ \\
\hline Initial & 20.0 & 500 & 50.0 & 100 & $4.32 \times 10^{-2}$ & 8.00 & 925 & 470 \\
Opt2kR & 24.1 & 557 & 62.3 & 110 & $3.76 \times \mathbf{1 0}^{-2}$ & 6.45 & 1171 & $\mathbf{4 2 9}$ \\
Opt2fz & 21.0 & 490 & 55.4 & 136 & $4.82 \times 10^{-2}$ & $\mathbf{8 . 3 3}$ & $\mathbf{1 1 2 9}$ & 438 \\
Opt2fR & 60.9 & 210 & 86.1 & 135 & 1.79 & $\mathbf{4 5 . 4}$ & 30.3 & $\mathbf{5 6 . 4}$ \\
Opt2zR & 27.2 & 569 & 57.7 & 111 & $3.99 \times 10^{-2}$ & 6.18 & $\mathbf{1 1 1 3}$ & $\mathbf{3 9 5}$ \\
\hline
\end{tabular}

From Table 3-Opt2kR, it can be noted that the non-controlled objective function $f$ decreases, which is undesirable, but $\Delta z$ increases (desirable).

From Table 3-Opt2fz, it can be noted that the non-controlled objective function $k$ increases, which is undesirable, but $R$ decreases (desirable).

From Table 3-Opt2fR, it can be noted that the non-controlled objective function $k$ increases (undesirable) and $\Delta z$ decreases (undesirable).

From Table 3-Opt2zR, it can be noted that the non-controlled objective function $k$ decreases (desirable) and $f$ decreases (undesirable).

\subsection{Tri-Objective Optimization Results}

The results of the tri-objective optimization are shown in Table 4. The values of the design variables and of the functions $(k, f, \Delta z, R)$ are shown. In particular, the minimized objective functions are highlighted in bold.

Table 4. Tri-objective optimization Opt3 results.

\begin{tabular}{ccccccccc}
\hline & $w[\mu \mathrm{m}]$ & $L_{\mathbf{1}}[\mu \mathrm{m}]$ & $L_{\mathbf{2}}[\mu \mathrm{m}]$ & $b[\mu \mathrm{m}]$ & $k\left[\mathrm{Nm}^{-\mathbf{1}}\right]$ & $f[\mathrm{kHz}]$ & $\Delta z[\mathrm{~nm}]$ & $R[\Omega]$ \\
\hline Initial & 20.0 & 500 & 50.0 & 100 & $\mathbf{4 . 3 2} \times \mathbf{1 0}^{-\mathbf{2}}$ & 8.00 & $\mathbf{9 2 5}$ & $\mathbf{4 7 0}$ \\
Final & 24.0 & 562 & 68.1 & 112 & $\mathbf{3 . 6 5} \times \mathbf{1 0}^{-2}$ & 6.33 & $\mathbf{1 2 2 6}$ & $\mathbf{4 2 9}$ \\
\hline
\end{tabular}


From Table 4, it can be noted that the non-controlled objective function $f$ decreases, which is undesirable. The results obtained with the tri-objective optimization are the most interesting ones, at least from the methodological viewpoint. However, we note that, depending on the designer's purpose and preferences, the results obtained with one or two objectives (Tables 2 and 3, respectively) could be preferred than the ones obtained with three objectives (Table 4). In general, in fact, the improvements achieved by means of a single- or bi-objective optimization could be substantial, even if the non-controlled objectives might undergo a modest deterioration. This is sometimes the case in engineering practice.

From the practical viewpoint, the solutions found in Opt2fz and Opt3 are felt to be the best ones, as explained in the following Section 3.2. Hence these cantilevers, as well as the initial cantilever, were fabricated and measured to verify the design and assess the optimization results.

\subsection{Measurements on Optimal Cantilevers}

The cantilevers according to the initial design as well as of the shapes obtained from Opt2fz and Opt 3 optimizations were considered to be optimal. With Opt2fz solution, significant increase in deflection and small decrease in resistance of loop were obtained. These two changes were most desirable and came without deterioration in the resonant frequency. Another solution considered to be optimal, the Opt3, exhibits even higher deflection and smaller resistance at the cost of $15 \%$ lower frequency. These two cantilevers were fabricated and finally measured. The example array is shown in Figure 5a. This manufactured cantilever array consists of four cantilevers, in particular:

- two cantilevers corresponding to initial design,

- $\quad$ final design, according to Opt2fz optimization result

- $\quad$ final design, according to Opt3 optimization result

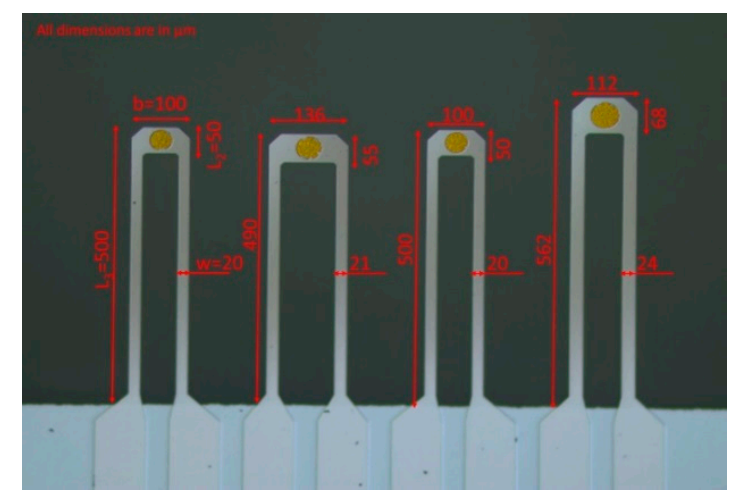

(a)

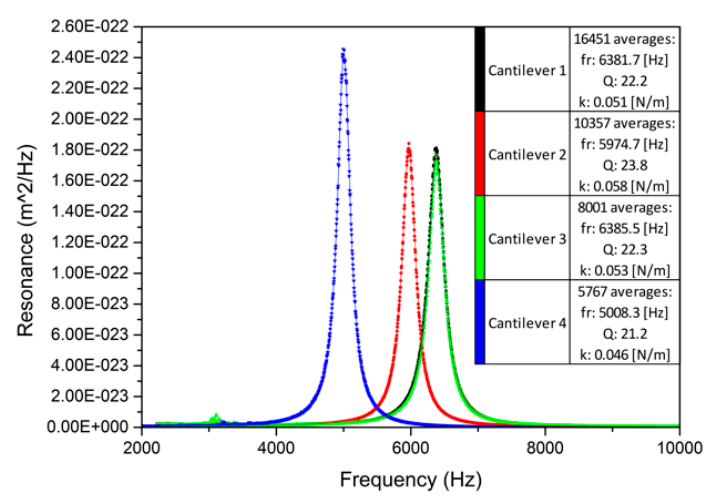

(b)

Figure 5. (a) example of manufactured cantilever array consisting of four cantilevers. Cantilevers 1 and 3 (from left to right) correspond to the initial design, while 2 and 4 are the optimal cantilevers after optimizations Opt2fz and Opt3. (b) the resonance response of the manufactured cantilevers.

To verify their properties, we have measured the thermomechanical noise of the cantilevers. The power spectrum of each resonant mode in the thermomechanical noise is defined by the following equation [28]:

$$
X_{t h}(f)=2 k_{B} T \pi^{-1} Q^{-1} f_{0}^{3} k^{-1}\left[\left(f_{0}^{2}-f^{2}\right)^{2}+f_{0}^{2} f^{2} Q^{-2}\right]^{-1},
$$

where $Q$ is the quality factor, $k_{B}$ is the Boltzmann constant, $T$ is the absolute temperature. By fitting parameters of Equation (11) to the measurements [18], the mechanical parameters are obtained. The thermomechanical noise formula is a result of the equipartition theorem [29]. Due to this theorem an object, which dissipates energy in thermal equilibrium, is subject to a fluctuation force. The measured resonance responses of the cantilevers shown in Figure 5a are shown in Figure 5b. 
Two cantilever arrays, each of which containing twice the same initial design cantilever, were fabricated. The measurement was then performed for both cantilever arrays, hence for four cantilevers (Table 5). This measurement provides information about the cantilever resonant frequency and its stiffness. Additionally, the resistance of the loop was measured. The cantilever provides direct electrical contacts. A Keithley 2000 multimeter was used to measure the resistance of the loop. The measurement was conducted in DC mode. The Keithley multimeter applies a $100 \mu \mathrm{A}$ current and subsequently the voltage drop was measured. The results of manufactured cantilever arrays according to initial design are shown in Table 5. The measured parameters of the 8 cantilevers of initial design vary at most by $8 \%$ from the mean values.

Table 5. Measurements on the initial cantilever.

\begin{tabular}{ccccccc}
\hline \multicolumn{3}{c}{ Measured Quantities } & \multicolumn{4}{c}{ Computed Quantities } \\
\hline $\boldsymbol{R}[\mathrm{k} \boldsymbol{\Omega}]$ & $\boldsymbol{f}[\mathrm{kHz}]$ & $\boldsymbol{Q}$ & $\boldsymbol{k}\left[\mathbf{N m}^{-\mathbf{1}}\right]$ & $\boldsymbol{F}_{\text {min }}[\mathrm{pN}]$ & $\boldsymbol{I}_{\text {min }}[\mathbf{n A}]$ & $\boldsymbol{P}_{\text {min }}[\mathbf{f W}]$ \\
\hline 1.89 & 6382 & 22.2 & 0.051 & 0.308 & 9.63 & 175 \\
2.26 & 6386 & 22.3 & 0.053 & 0.313 & 9.79 & 217 \\
1.88 & 6633 & 23.4 & 0.060 & 0.319 & 9.98 & 187 \\
2.26 & 6634 & 23.4 & 0.055 & 0.306 & 9.55 & 206 \\
1.88 & 6461 & 22.9 & 0.057 & 0.319 & 9.96 & 187 \\
2.26 & 6502 & 22.9 & 0.056 & 0.315 & 9.84 & 219 \\
1.88 & 6787 & 23.9 & 0.060 & 0.312 & 9.76 & 179 \\
2.26 & 6799 & 24.6 & 0.064 & 0.318 & 9.93 & 223 \\
\hline
\end{tabular}

The cantilever parameters manufactured according to Opt2fz and Opt 3 optimization results are provided in Table 6.

Table 6. Measurements on the Opt2fz and Opt3 cantilever.

\begin{tabular}{cccccccc}
\hline \multicolumn{4}{c}{ Measured Quantities } & \multicolumn{3}{c}{ Computed Quantities } \\
\hline & $\boldsymbol{R}[\mathbf{k} \boldsymbol{\Omega}]$ & $f[\mathbf{k H z}]$ & $\boldsymbol{Q}$ & $\boldsymbol{k}\left[\mathbf{N m}^{-\mathbf{1}}\right]$ & $\boldsymbol{F}_{\boldsymbol{\text { min }}}[\mathbf{p N}]$ & $\boldsymbol{I}_{\boldsymbol{m i n}}[\mathbf{n A}]$ & $\boldsymbol{P}_{\text {min }}$ [fW] \\
\hline Opt2fz, array1 & 2.16 & 5975 & 23.8 & 0.058 & 0.328 & 7.13 & 110 \\
Opt2fz, array2 & 2.15 & 5819 & 25.3 & 0.063 & 0.336 & 7.30 & 115 \\
Opt3, array1 & 1.74 & 5008 & 21.2 & 0.046 & 0.338 & 9.60 & 160 \\
Opt3, array2 & 1.74 & 5205 & 22.3 & 0.038 & 0.294 & 8.35 & 121 \\
\hline
\end{tabular}

\subsection{Electromagnetic Actuation of the Cantilevers}

One of the manufactured arrays was subject to electromagnetic actuation. The array was placed in a uniform magnetic field of $317 \mathrm{mT}$. Then the amplitude of vibration vs actuating current characteristics were recorded. Such experiment was performed for each cantilever in the array. During the experiment the current was flowing only through one of the cantilevers at a time. Figure 6 contains the results of the electromagnetic actuation characterisation. The actuation was performed in resonance and in low frequency region $(100-1000 \mathrm{~Hz})$ - so called quasi static characteristics. It can be noted that under the same conditions, the manufactured cantilevers of OPT2fz and OPt3 design exhibit significantly higher vibration amplitudes $\Delta z$. 


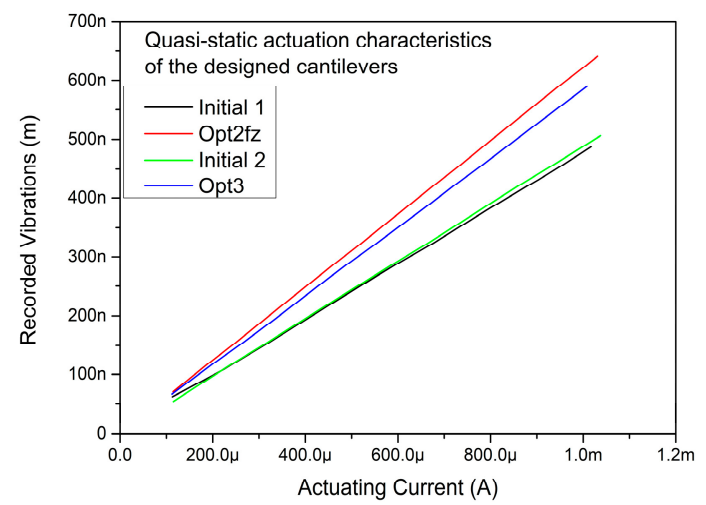

(a)

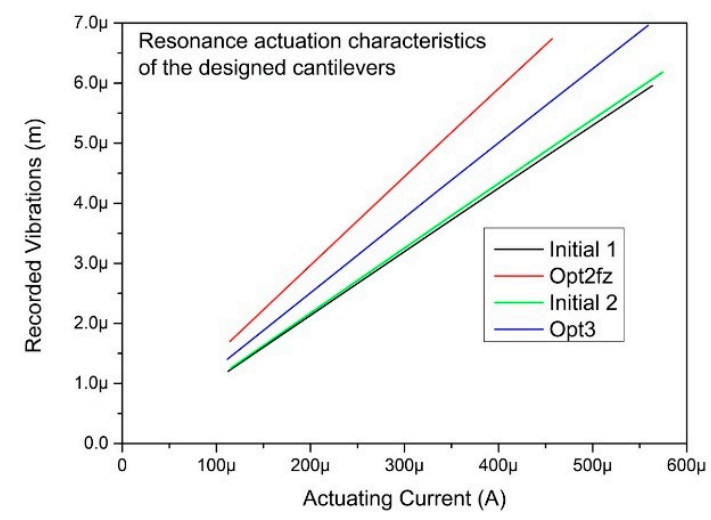

(b)

Figure 6. Results of the electromagnetic actuation of the optimal cantilevers (a) results recorded in the low frequency region $(100-1000 \mathrm{~Hz})$ and $(\mathbf{b})$ in resonance. The experiments were performed under uniform $317 \mathrm{mT}$ magnetic field.

\section{Discussion}

The measured results of the initial cantilever differ slightly from those calculated for the initial prototype in Tables 2-4. In fact, the manufactured cantilevers contain the gold layer at each end. This gold is supposed to improve reflection of the laser beam from cantilever surface in AFM measurements. The resonant frequency differs due to the mass of the added gold layer. The resulting frequency of the manufactured cantilevers is lower and equal to approximately $6.5 \mathrm{kHz}$. It should be also noted that, due to the many technological processes required to manufacture the cantilevers, some drifts in measured parameters are unavoidable. This can be particularly seen for the initial cantilever, where we can compare results for four manufactured pieces and the parameters of each of them differ.

The optimization procedure could account for the mass of the gold layer at the cantilever end. In fact, the gold layer is contained within small area at the end and therefore, it can be treated as a point mass load. This leads to a possibility for the correction of the resonant frequency, using the formula:

$$
f=\frac{\sqrt{k}}{m_{e}+m_{g}}
$$

where $m_{e}$ is the effective mass of the cantilever, $m_{g}$ is the mass of gold. The mass of the gold layer could be assessed from the volume.

The main difference between the optimization results and manufactured cantilevers is the value of the resistance. It comes out from the fact that the value of doping concentration in the manufactured cantilevers is lower than the assumed one in the optimization procedure. However, this discrepancy does not affect the optimization results and its assessment (as it only influences material properties).

Provided that all the optimization runs start from the same initial point, the following remarks can be put forward:

The solutions obtained in Opt1k, Opt1f, Opt1z and Opt1R differ in both design vector $\left(w, L_{1}, L_{2}, b\right)$ and objective vector $(k, f, \Delta z, R)$. This proves that a single solution simultaneously satisfying all the design criteria does not exist. From the optimization theory viewpoint, it is a design conflict problem that can be studied via Pareto optimality.

Solutions obtained in Opt2 and Opt3 can be considered Pareto-equivalent to the initial one, because three objectives improve, while one objective deteriorates (e.g., solution of Opt 3 where $k$, $\Delta z$ and $R$ improve, while $f$ deteriorates). From the application viewpoint, provided the amount of deterioration in one objective is acceptable, Pareto-equivalent solutions may represent a good alternative to the initial solution. 
From the methodological point of view, it can be stated that the applied evolutionary algorithm is suitable for solving the shape optimization of cantilevers. In particular, it can be stated that this method works well in finding an improved cantilever-based device, when dealing with design problems characterized by less than ten design variables and up to three objective functions. In general, this method can be used for a class of optimal shape design problems exhibiting the same complexity in terms of number of design variables and objective functions.

\section{Conclusions}

A class of electromagnetically actuated cantilevers has been considered. Their optimal shape design has been carried out by means of a multi-objective design method, based on evolutionary algorithm. The subsequent fabrication of cantilever arrays and relevant measurements have assessed the optimization results. This puts the ground for a more general procedure of cantilever design for nanometrology purposes.

Author Contributions: P.D.B. conceived and formulated the optimal design problem, run the optimization procedure and analyzed the results. T.G. wrote the introduction of the article. K.O. and W.M. measured the cantilevers and analysed the measurement data. They also prepared the measurement results chapter. M.E.M. contributed to the analysis of the optimization results and the discussion. She also wrote the paper. S.W. read subsequent versions of the paper and contributed to the bibliographic references. A.S. prepared the technological process and manufactured the cantilevers. He was also responsible for preparing chapter of the manufacturing process.

Funding: The fabrication and measurements of the electromagnetic actuation were done within the NCN OPUS 9 Grant-“Metrology of molecular interactions using electromagnetically actuated MEMS force sensors-MetMolMEMS" (Grant No. 2015/17/B/ST7/03876).

Conflicts of Interest: The authors declare no conflict of interest.

\section{References}

1. Lang, H.P.; Hegner, M.; Gerber, C. Nanomechanical cantilever array sensors. In Springer Handbook of Nanotechnology; Springer: Berlin/Heidelberg, Germany, 2013; pp. 457-485.

2. Gaspar, J.; Chu, V.; Conde, J.P. Electrostatic actuation of thin-film microelectromechanical structures. J. Appl. Phys. 2003, 93, 10018-10029. [CrossRef]

3. Minne, S.C.; Manalis, S.R.; Quate, C.F. Parallel atomic force microscopy using cantilevers with integrated piezoresistive sensors and integrated piezoelectric actuators. Appl. Phys. Lett. 1995, 67. [CrossRef]

4. Angelov, T.; Roeser, D.; Ivanov, T.; Gutschmidt, S.; Sattel, T.; Rangelow, I.W. Thermo-mechanical transduction suitable for high-speed scanning probe imaging and lithography. Microelectron. Eng. 2016, 154, 1-7. [CrossRef]

5. Woszczyna, M.; Zawierucha, P.; Pałetko, P.; Zielony, M.; Gotszalk, T.; Sarov, Y.; Ivanov, T.; Frank, A.; Zöllner, J.-P.; Rangelow, I.W. Micromachined scanning proximal probes with integrated piezoresistive readout and bimetal actuator for high eigenmode operation. J. Vac. Sci. Technol. B 2010, 28. [CrossRef]

6. Majstrzyk, W.; Ahmad, A.; Ivanov, T.; Reum, A.; Angelow, T.; Holz, M.; Gotszalk, T.; Rangelow, I.W. Thermomechanically and electromagnetically actuated piezoresistive cantilevers for fast-scanning probe microscopy investigations. Sens. Actuators A Phys. 2018, 276, 237-245. [CrossRef]

7. Nieradka, K.M.; Kopiec, D.; Małozięć, G.; Kowalska, Z.; Grabiec, P.; Janus, P.; Sierakowski, A.; Domański, K.; Gotszalk, T. Fabrication and characterization of electromagnetically actuated microcantilevers for biochemical sensing, parallel AFM and nanomanipulation. Microelectron. Eng. 2012, 98, 676-679. [CrossRef]

8. Shen, B.; Allegretto, W.; Hu, M.; Robinson, A. Cmos micromachined cantilever-in-cantilever devices with magnetic actuation. IEEE Electron Device Lett. 1996, 17, 372-374. [CrossRef]

9. Adhikari, R.; Kaundal, R.; Sarkar, A.; Rana, P.; Das, A.K. The cantilever beam magnetometer: A simple teaching tool for magnetic characterization. Am. J. Phys. 2012, 80, 225. [CrossRef]

10. Hsieh, C.H.; Dai, C.L.; Yang, M.Z. Fabrication and characterization of cmos-mems magnetic microsensors. Sensors 2013, 13, 14728-14738. [CrossRef] [PubMed]

11. Rhoads, J.F.; Kumar, V.; Shaw, S.W.; Turner, K.L. The non-linear dynamics of electromagnetically actuated microbeam resonators with purely parametric excitations. Int. J. Non-Linear Mech. 2013, 55, 79-89. [CrossRef] 
12. Somnath, S.; Liu, J.O.; Bakir, M.; Prater, C.B.; King, W.P. Multifunctional atomic force microscope cantilevers with Lorentz force actuation and self-heating capability. Nanotechnology 2014, 25. [CrossRef] [PubMed]

13. Majstrzyk, W.; Mognaschi, M.E.; Orłowska, K.; Di Barba, P.; Sierakowski, A.; Dobrowolski, R.; Grabiec, P.; Gotszalk, T. Electromagnetic cantilever reference for the calibration of optical nanodisplacement systems. Sens. Actuators A Phys. 2018. under revision.

14. Fritz, J.; Baller, M.K.; Lang, H.P.; Strunz, T.; Meyer, E.; Güntherodt, H.J.; Delamarche, E.; Gerber, C.; Gimzewski, J.K. Stress at the solid-liquid interface of self-assembled monolayers on gold investigated with a nanomechanical sensor. Langmuir 2000, 16. [CrossRef]

15. Kopiec, D.; Pałetko, P.; Nieradka, K.; Majstrzyk, W.; Kunicki, P.; Sierakowski, A.; Jóźwiak, G.; Gotszalk, T. Closed-loop surface stress compensation with an electromagnetically actuated microcantilever. Sens. Actuators B Chem. 2015, 228, 62-68. [CrossRef]

16. Di Barba, P.; Mognaschi, M.E.; Venini, P.; Wiak, S. Biogeography-inspired multiobjective optimization for helping MEMS synthesis. Arch. Electr. Eng. 2017, 66, 607-623. [CrossRef]

17. Tortonese, M.; Barrett, R.C.; Quate, C.F. Atomic resolution with an atomic force microscope using piezoresistive detection. Appl. Phys. Lett. 1993, 62. [CrossRef]

18. Walters, D.A.; Cleveland, J.P.; Thomson, N.H.; Hansma, P.K.; Wendman, M.A.; Gurley, G.; Elings, V. Short cantilevers for atomic force microscopy. Rev. Sci. Instrum. 1996, 67. [CrossRef]

19. Di Barba, P. Multiobjective Shape Design in Electricity and Magnetism; Springer-Verlag: Berlin, Germany, 2010.

20. Di Barba, P.; Savini, A.; Wiak, S. Field Models in Electricity and Magnetism; Springer: Berlin, Germany, 2008.

21. Di Barba, P.; Mognaschi, M.E.; Lowther, D.A.; Sykulski, J.K. A benchmark TEAM problem for multi-objective pareto optimization of electromagnetic devices. IEEE Trans. Magn. 2018, 54. [CrossRef]

22. Di Barba, P.; Mognaschi, M.E. Industrial design with multiple criteria: Shape optimization of a permanent-magnet generator. IEEE Trans. Magn. 2009, 45, 1482-1485. [CrossRef]

23. Costamagna, E.; Di Barba, P.; Mognaschi, M.E.; Savini, A. Fast algorithms for the design of complex-shape devices in electromechanics. In Studies in Computational Intelligence; Springer: Berlin, Germany, 2010; Volume 327, pp. 59-86.

24. Gotszalk, T.; Grabiec, P.; Rangelow, I.W. Piezoresistive sensors for scanning probe microscopy. Ultramicroscopy 2000, 82, 39-48. [CrossRef]

25. Buguin, A.; Du Roure, O.; Silberzan, P. Active atomic force microscopy cantilevers for imaging in liquids. Appl. Phys. Lett. 2001, 78. [CrossRef]

26. Węrzecki, M.; Wolski, D.; Bar, J.; Budzyński, T.; Chłopik, A.; Grabiec, P.; Kłos, H.; Panas, A.; Piotrowski, T.; Słysz, W.; et al. 64-element photodiode array for scintillation detection of x-rays. In Proceedings of the 13th International Scientific Conference on Optical Sensors and Electronic Sensors, Lodz, Poland, 19 August 2014.

27. Rueda, H.A.; Law, M.E. Modeling of Strain in Boron-Doped Silicon Cantilevers. In Proceedings of the International Conference on Modeling and Simulation of Microsystems, Sinaia, Romania, 6-10 October 1998.

28. Stark, R.W.; Drobek, T.; Heckl, W.M. Thermomechanical noise of a free v-shaped cantilever for atomic-force microscopy. Ultramicroscopy 2001, 86, 207-215. [CrossRef]

29. Butt, H.J.; Jaschke, M. Calculation of thermal noise in atomic force microscopy. Nanotechnology 1995, 6, 1-7. [CrossRef]

(C) 2018 by the authors. Licensee MDPI, Basel, Switzerland. This article is an open access article distributed under the terms and conditions of the Creative Commons Attribution (CC BY) license (http://creativecommons.org/licenses/by/4.0/). 